\title{
STRAIN RATES IN GREECE USING GPS MEASUREMENTS FROM 1994-2000
}

\author{
Rontogianni S. ${ }^{1}$, Clarke P. ${ }^{1}$, King M. ${ }^{1}$, Lavallée D. ${ }^{1}$, England P. ${ }^{2}$, Parsons B. ${ }^{2}$, \\ and Floyd $\mathbf{M}^{2}$ \\ ${ }^{1}$ University of Newcastle, School of Civil Engineering and Geosciences, UK, \\ Sofia.Rontogianni@ncl.ac.uk,Peter.Clarke@newcastle.ac.uk,M.A.King@newcastle.ac.uk, \\ D.A.Lavallee@newcastle.ac.uk. \\ 2 University of Oxford, Department of Earth Sciences, UK, Philip.England@earth.ox.ac.uk \\ Barry.Parsons@earth.ox.ac.uk Michael.Floyd@earth.ox.ac.uk
}

\begin{abstract}
Fifteen Greek GPS campaigns from 1994-2001 are reprocessed in ITRF2000 using Bernese v4.2 software. The reference frame is navigated by 43 global, long running IGS sites, which are included in the coordinate final solution together with $\sim 280$ local sites. A loosely constrained solution is derived. We combine the 221 daily SINEX files in a least squares approach with the TANYA software, producing the coordinate time series and the velocity field in a European Fixed reference frame. Sites around Chalkidiki and Grevena show a residual motion $\sim 10 \mathrm{~mm} / \mathrm{yr}$. Sites around Attica, Evvia, SE Peloponnisos, Aegean Islands have a SW residual motion reaching $\sim 40 \mathrm{~mm} / \mathrm{yr}$ in South Peloponnisos. The Patras area shows a more southwesterly motion with respect to the rest of the sites, moving $\sim 30-35 \mathrm{~mm} / \mathrm{yr}$.

Geodetic strain is derived by two methods: by discrete estimation within contiguous polygons, and by fitting a continuous minimum curvature surface to the velocity field.
\end{abstract}

Key words: GPS, Greece, Velocity Field, Strain, Seismic Hazard.

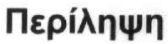

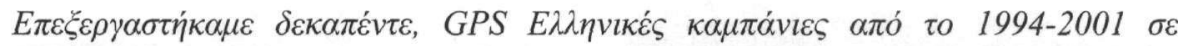

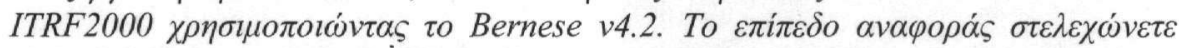

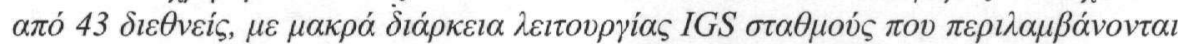

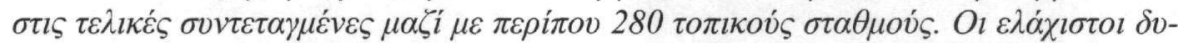

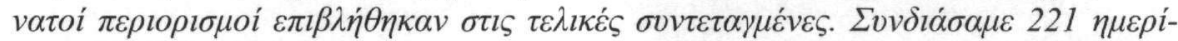

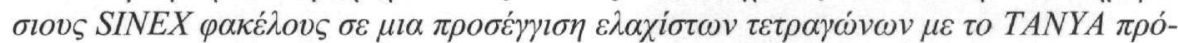

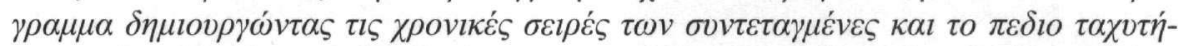

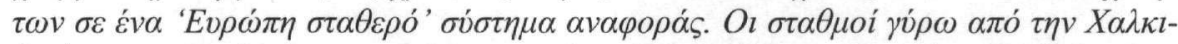

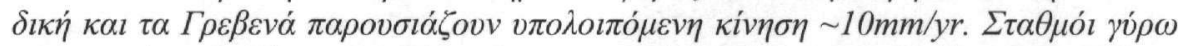

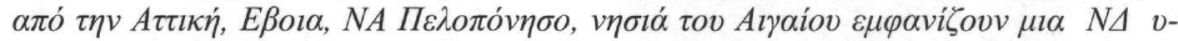

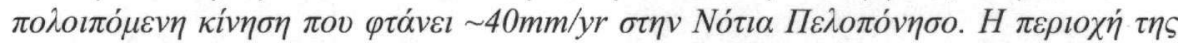

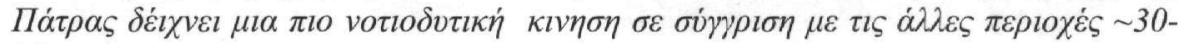
$35 m m / y r$. 


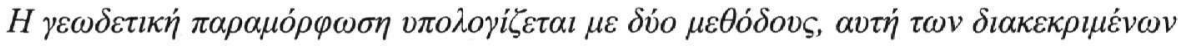

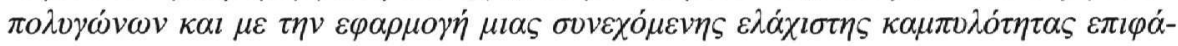

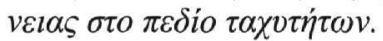

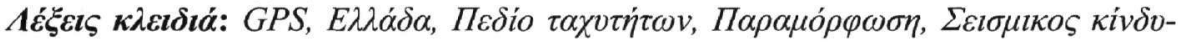
vos.

\section{Introduction}

The Eastern Mediterranean has been identified as an ideal natural laboratory for studying the kinematics and dynamics of plate interactions (Plag et al. 1998). It is one of the most tectonically active regions of Earth's surface (McKenzie 1972, McKenzie 1978) as is demonstrated by its seismicity (Ambraseys 2001, Ambraseys and Jackon 1997, Ambraseys and Jackson 1990). The region is on a convergent plate boundary comprising the subduction of the African Plate under the Eurasian plate while the Arabian plate approaches the Eurasian plate in a northwestward motion. That motion leads the Anatolia plate (defined as the region of Turkey between the North and East Anatolian Faults) to be displaced to the west and rotate in a counterclockwise sense relative to Eurasia (Reilinger et al. 1997).

The Hellenic area (Fig. 1) has been the subject of several studies that worked either with earthquake fault plane solution data or with geodetic data in an attempt to solve the tectonic puzzle that surrounds this highly deformed area. Deep seismicity under the sea of Crete is related to the subduction of the northward moving (relative to Eurasia) African oceanic lithosphere, whereas shallow seismicity throughout the Aegean Sea and mainland Greece is related to the southwestward (again relative to Eurasia) extension of the Aegean continental lithosphere driven by a combination of gravitational spreading of the Anatolian continental lithosphere from the east

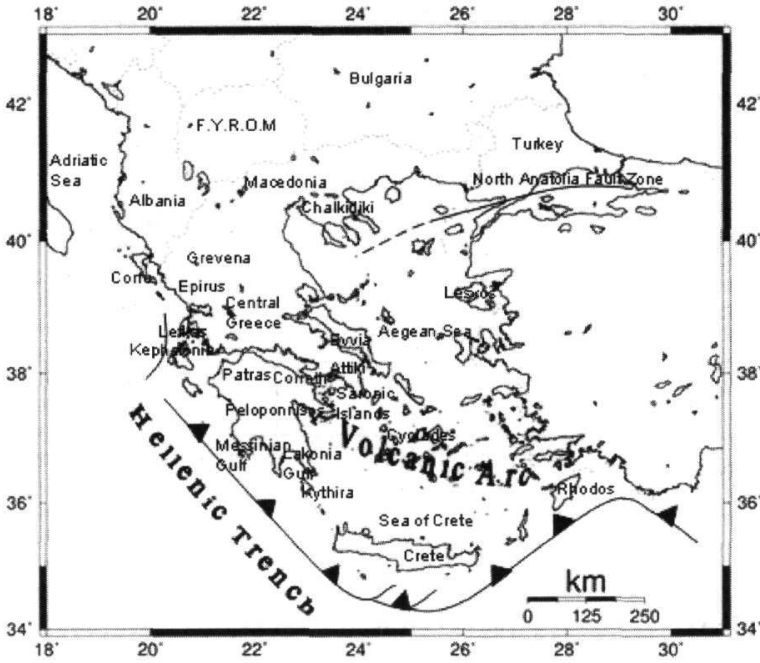

Figure 1-Main features of the Hellenic area and location map

and roll-back of the subducting African plate to the south (Le Pichon and Angelier 1979, Papazachos 1990). The seismicity to the north along the coast of former Yugoslavia, Albania and NW Greece defines the eastern boundary of the 'Adriatic block' which shows intermediate behaviour between that of the European and African plates. The convergence between the Apulian platform and the Hellenides mountains, the Albanides and the Dinarides is a continental type collision. The lithosphere of the Ionian basin is probably oceanic and the convergent motion results in subduction beneath the Hellenic Trench. The transition between the active lithospheric subduction and the continental collision is located around the Ionian Islands. (Papazachos and Kiratzi 1996)

Multiple kinematic (Armijo et al. 1996, McKenzie 1970, McKenzie 1978, McKenzie and Jackson 1983, McKenzie and Jackson 1986, Nyst and Thatcher 2004, Taymaz et al. 1991, Thatcher 2003) and dynamic models (Goldsworthy et al. 2002, Hatzfeld et al. 1997, Kreemer et al. 2003, Mantovani et al. 2000, Meijer and Wortel 1997) have been proposed as an attempt to describe the observed present-day deformation highlighting many important features of the Hellenic and surrounding areas (Anatolia, western Turkey) but not developing a single, widely-accepted, consistent model. Hence the geological questions that still need answering in the light of the latest data are. 
How can the Hellenic area be divided into zones of different deformational style and rate? What amount of geodetic strain is released by seismicity? How important is aseismic deformation?

\section{GPS data}

The complex tectonics and high deformation rates in the Hellenic area have attracted the curiosity of numerous GPS groups who have organised campaigns and designed networks to assess the strain accumulation throughout this area (Briole et al. 2000, Hollenstein et al. 2005, Kahle et al. 2000, Kahle et al. 1999, McClusky et al. 2000). This has resulted in a number of overlapping GPS networks. Networks were also designed around Greece in order to strengthen links between sites and fill in areas not previously occupied, in the HELLENET and the SING projects (Cruddace et al. 1999).

Table 1 - GPS campaigns used during the project

\begin{tabular}{|c|c|c|c|c|c|}
\hline Network & Region & $\begin{array}{l}\text { Cam- } \\
\text { paign/Software }\end{array}$ & Year & $\begin{array}{l}\text { Day of } \\
\text { year (DOY) }\end{array}$ & $\begin{array}{l}\text { No. of local } \\
\text { sites }\end{array}$ \\
\hline Aegean & Aegean & $\begin{array}{l}\text { Aegean94,Bernese } \\
\text { v } 4.2\end{array}$ & 1994 & $255-272$ & 4 \\
\hline Aegean & Aegean & $\begin{array}{l}\text { Aegean96,Bernese } \\
\text { v4.2 }\end{array}$ & 1996 & $252-271$ & 4 \\
\hline SING & Argos & $\begin{array}{l}\text { Argos } 98, \text { Bernese } \\
\text { v4.2 }\end{array}$ & 1998 & $179-198$ & 48 \\
\hline SING & Patras & $\begin{array}{l}\text { Patra98,Bernese } \\
\text { v4.2 }\end{array}$ & 1998 & $284-297$ & 28 \\
\hline SING & $\begin{array}{l}\text { Chalkidiki, } \\
\text { S Peloponnisos }\end{array}$ & $\begin{array}{l}\text { Chasp98,Bernese } \\
\text { v4.2 }\end{array}$ & 1998 & $263-275$ & 50 \\
\hline SING & Attiki & $\begin{array}{l}\text { Attiki99,Bernese } \\
\text { v } 4.2\end{array}$ & 1999 & $276-285$ & 12 \\
\hline SING & $\begin{array}{l}\text { Argos, Patras, } \\
\text { S Peloponnisos }\end{array}$ & $\begin{array}{l}\text { Paspar99,Bernese } \\
\text { v } 4.2\end{array}$ & 1999 & $152-163$ & 54 \\
\hline SING & Chalkidiki & $\begin{array}{l}\text { Chalkidiki99,Berne } \\
\text { se v4.2 }\end{array}$ & 1999 & $283-295$ & 36 \\
\hline SING & Argos, Patras & $\begin{array}{l}\text { Argpa00,Bernese } \\
\text { v } 4.2\end{array}$ & 2000 & $184-189$ & 32 \\
\hline SING & Chalkidiki & $\begin{array}{l}\text { Chalkidiki00,Berne } \\
\text { se v } 4.2\end{array}$ & 2000 & $272-276$ & 30 \\
\hline SING & Corinth & $\begin{array}{l}\text { Corinth00,Bernese } \\
\text { v4.2 }\end{array}$ & 2000 & 091-195 & 19 \\
\hline Grevena & Grevena & $\begin{array}{l}\text { Grevena96,Bernese } \\
\text { v4.2 }\end{array}$ & 1996 & $128-160$ & 41 \\
\hline Grevena & Grevena & $\begin{array}{l}\text { Grevena98,Bernese } \\
\text { v4.2 }\end{array}$ & 1998 & $153-178$ & 59 \\
\hline Grevena & Grevena & $\begin{array}{l}\text { Grevena00,Bernese } \\
\text { v4.2 }\end{array}$ & 2000 & $245-254$ & 55 \\
\hline Hellenet & Hellenet & $\begin{array}{l}\text { Hellenet97,Bernese } \\
\text { v } 4.2\end{array}$ & 1997 & $252-274$ & 72 \\
\hline Egion & Egion & $\begin{array}{l}\text { Egion95a,(Jun), } \\
\text { GAMIT v10.2 }\end{array}$ & 1995 & $171-176$ & 40 \\
\hline Egion & Egion & $\begin{array}{l}\text { Egion95b,(Sep), } \\
\text { GAMIT v10.2 }\end{array}$ & 1995 & $276-281$ & 56 \\
\hline Egion & Egion & $\begin{array}{l}\text { Egion96,(Jun), } \\
\text { GAMIT v10.2 }\end{array}$ & 1996 & $135-153$ & 60 \\
\hline TOTAL & & & & & 280 \\
\hline
\end{tabular}


Although the majority of these campaign datasets have been analysed and reported previously, it has not been possible to combine them rigorously into a single velocity field because they have been processed using different observation models, constraints, and reference frames. Full variance-covariance information is not always present from previous solutions. Effort made by the researchers to remove the existing constraints was not successful, leading each group to extract its own individual solutions.

Knowing about this drawback we decided to reprocess 18 previous campaigns spanning from 1994-2000 including around 280 local sites (as well as data from the "DION G" GPS pillar in Greece provided by the National Observatory of Athens), combine them and obtain a unified solution for the Greek area (Table 1). We did not work towards removing a co-seismic model from our dataset because the only data from before the 1995 Egion earthquake that we have used are the Aegean campaign (sites ASKT, DION, KATV, XRIS). These are sites distant from the event and so there is no need to remove a co-seismic model.

To produce a better reference frame definition, the frame of each campaign was navigated from as many as 43 IGS and EUREF sites. These sites were chosen to have a global distribution and to have been in continuous operation since 1994 (Fig. 2). The RINEX files for all of these stations were obtained from the Scripps Orbit and Permanent Array Center (SOPAC) http://sopac.ucsd.edu/dataArchive.

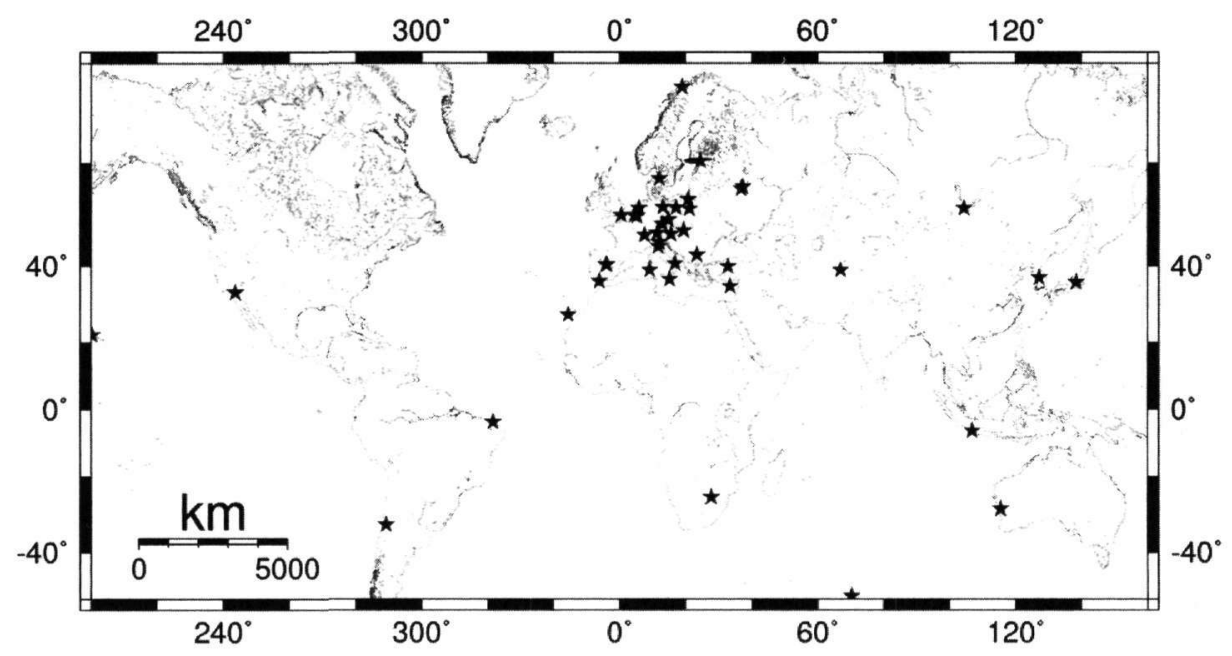

Figure 2 -The 43 Global IGS and EUREF sites used in this project

The raw data were imported in the software via the RINEX (Gurtner 1994) format. Where necessary, transformation from raw (Trimble or Ashtech) format took place using UNAVCO' $s$ TEQC program (Estay and Meertens 1999, UNAVCO). The information given in the RINEX header was corrected in order to agree with the log files. All antenna heights were corrected to agree with the standard ARP (antenna reference point) adopted by IGS. Because the daily networks typically had a mixture of antenna types, we used elevation-dependent phase centre offsets as provided by NOAA (http://www.ngs.noaa.gov/ANTCAL).

\section{GPS data processing}

For the majority of data processing the Bernese 4.2 GPS software (Beutler et al. 2001) was used. As a reference frame for the whole evaluation we used ITRF2000, IGS precise orbits, and we took under consideration the troposphere, ionosphere, and ocean tide loading effects as described in Table 2 . We corrected any detected cycle slips, and then attempted to resolve all carrier phase ambiguities to integers as part of each daily network solution, typically with more than $70 \%$ success. 
The three Egion campaigns were processed in the GAMIT version 10.2 software using a similar strategy but with sessions aligned to the local campaign observations rather than the UT day.

For each campaign, the normal equations of daily sessions were generated and combined into a campaign solution as a check on processing. The daily SINEX files were also produced and saved in order to be used in velocity analysis. For the IGS sites, the a priori velocities were given in ITRF2000 and free network conditions were set up for their coordinates. Table 2 summarises the processing strategy followed.

Table 2 - Processing strategy followed during the project

\begin{tabular}{|l|l|}
\hline Session choice & "Day-by-day" strategy, strictly aligned to UT days \\
\hline Baseline choice & $\begin{array}{l}\text { Minimise total baseline length and antenna mismatches, whilst maximising } \\
\text { common observation times. }\end{array}$ \\
\hline Orbits & IGS precise orbits, held fixed. \\
\hline Data windowing & $10^{\circ}$ elevation mask (unless higher in raw data). \\
\hline Ionosphere model & $\begin{array}{l}\text { Ionosphere free observable used; CODE global ionosphere map used to aid } \\
\text { ambiguity resolution in wide-lane solutions. }\end{array}$ \\
\hline Troposphere model & $\begin{array}{l}\text { Saastamoinen model for dry zenith delay; wet zenith delay estimated at 2- } \\
\text { hour intervals. Niell mapping function (Niell 1996) (wet and dry). }\end{array}$ \\
\hline Tidal loading & $\begin{array}{l}\text { IERS 1996 Conventions for solid Earth tides incorporated within Bernese } \\
\text { software. Ocean tide loading specified a priori for 11 major constituents us- } \\
\text { ing the CSR4.0 ocean tide model, computed using } \\
\text { http://www.oso.chalmers.se/ loading/ }\end{array}$ \\
\hline Ambiguity resolution & $\begin{array}{l}\text { Double-difference ambiguities resolved on per-baseline basis within daily } \\
\text { network solutions, using either "Quasi Ionosphere Free" model (campaigns } \\
\text { Grevena 96, Patras 98, Argos 98) or SIGMA-dependent rounding using a } \\
\text { wide-lane followed by ionosphere-free solution (all other campaigns). }\end{array}$ \\
\hline
\end{tabular}

\section{Velocity field results}

Site velocities have been estimated from the combination of 221 loosely constrained daily SINEX files that were created in the Bernese 4.2 processing stage, using the TANYA software (Davies 1997, Davies and Blewitt 2000, Lavallée 2000). Station daily coordinates are rigorously combined using a free network approach (Davies 1997, Davies and Blewitt 2000). In our case the solution coming out from Bernese 4.2 is a loosely constrained solution, and any constraints on the geometry are applied from the fixed IGS orbits. The core IGS00 SINEX file (the IGS realisation of ITRF2000 Core) was used (Altamimi et al. 2002) to navigate the solution to the ITRF2000 reference frame. Antenna height corrections and a three dimensional data-snooping outlier rejection method are also applied if needed at this stage.

The sites were gradually introduced in the solution starting with the ones that had more than 50 observations (the majority of the IGS sites). All sites that were present in more than one campaign and had two or more observations were used in the final solution. An iterative residual outlier rejection boundary of four standard deviations was used to exclude outliers in each station coordinate residual time series. The resulting free network solution is aligned to ITRF2000 by applying 12 Helmert transformations ( 7 Helmert parameters plus their time derivatives but no scale or scale rate). From the time series of the daily solutions we estimate a kinematic solution which includes a reference epoch position (1999.00) and a constant velocity for each station.

For the sites whose time series were edited, no more than $10 \%$ of the overall number of observations were removed. Excluded from the solution are also some of the IGS sites that had very noisy time series The majority of excluded IGS sites are located in the southern hemisphere (i.e. very long baselines, with not enough overlap of visible satellites between the two stations creating the baseline). Excluded sites are YAR1, USUD, SUWN, KOKB, PIN2, KERG, HARK, IRKT. Data for the station ANKR are used until 1999, i.e. before the devastating 1999 Izmit earthquake (17 
August $\mathrm{M}_{\mathrm{S}}$ 7.4) that took place in this area. No other sites suffered from discernible co-seismic offsets.

The solution in ITRF2000 was expressed in a European Fixed Reference frame by applying the Altamimi et al. (2002) absolute Euler pole for Eurasia (Table 3).

Table 3 - Site residuals to the Eurasian absolute Euler pole from Altamimi et al. (2002). The IGS and EUREF sites are given in blue

\begin{tabular}{|c|c|c|c|c|c|c|}
\hline SITE & Latitude & Longitude & $\begin{array}{r}\text { Residuals } \\
\text { North } \\
m m / y r\end{array}$ & $\begin{array}{r}\text { Residuals } \\
\text { East } \\
m m / y r\end{array}$ & $\begin{array}{c}\sigma-n o r t h \\
m m / y r\end{array}$ & $\begin{array}{c}\sigma-e a s t \\
m m / y r\end{array}$ \\
\hline $\mathrm{AD} 18$ & 38.324 & 22.184 & -11.942 & -18.979 & 1.150 & 1.150 \\
\hline AD22 & 38.356 & 22.240 & -6.494 & -25.693 & 1.330 & 1.380 \\
\hline AETS & 37.244 & 21.835 & -32.503 & -25.888 & 0.360 & 0.400 \\
\hline AGFA & 37.756 & 24.906 & -35.712 & -19.086 & 0.720 & 0.600 \\
\hline AGMA & 38.166 & 24.065 & -25.638 & -15.331 & 0.330 & 0.300 \\
\hline AGNI & 37.669 & 22.488 & -38.244 & -14.597 & 0.520 & 0.470 \\
\hline AIOL & 37.966 & 24.751 & -38.105 & -16.042 & 3.360 & 5.970 \\
\hline ANKR & 39.887 & 32.758 & -2.216 & -22.700 & 0.110 & 0.100 \\
\hline ARIO & 38.327 & 21.766 & -5.400 & 4.519 & 0.540 & 0.600 \\
\hline AS76 & 38.674 & 22.441 & -9.275 & -16.283 & 1.460 & 1.460 \\
\hline ASKT & 40.928 & 25.566 & -5.345 & 9.686 & 0.140 & 0.130 \\
\hline ASTP & 36.586 & 26.406 & -24.371 & 9.971 & 0.650 & 0.670 \\
\hline ATKO & 38.494 & 21.120 & -19.418 & 5.788 & 0.480 & 0.410 \\
\hline AY88 & 39.994 & 21.743 & -11.889 & -3.148 & 0.120 & 0.120 \\
\hline BOR1 & 52.277 & 17.073 & 0.647 & 0.609 & 0.100 & 0.090 \\
\hline BRUS & 50.798 & 4.359 & 0.384 & -0.947 & 0.090 & 0.100 \\
\hline CAGL & 39.136 & 8.973 & 0.762 & -0.315 & 0.090 & 0.110 \\
\hline CG05 & 39.361 & 23.186 & -21.231 & -6.483 & 0.370 & 0.230 \\
\hline CG19 & 38.781 & 22.448 & -14.698 & -24.597 & 1.800 & 1.930 \\
\hline CG20 & 38.651 & 22.623 & -11.360 & -24.721 & 1.960 & 2.110 \\
\hline CG22 & 38.640 & 22.397 & -15.381 & -21.988 & 1.900 & 1.970 \\
\hline CG30 & 38.397 & 22.142 & -16.352 & -15.099 & 0.180 & 0.180 \\
\hline CG31 & 38.348 & 22.285 & -14.911 & -12.221 & 2.570 & 2.460 \\
\hline CG32 & 38.399 & 22.577 & -14.799 & -24.606 & 1.780 & 1.660 \\
\hline CG33 & 38.428 & 22.869 & -23.832 & -17.744 & 3.500 & 3.400 \\
\hline CG42 & 38.228 & 21.973 & -29.680 & -16.217 & 0.350 & 0.360 \\
\hline CG43 & 38.130 & 22.192 & -25.240 & -27.161 & 1.800 & 1.850 \\
\hline CG47 & 38.021 & 22.945 & -30.608 & -14.920 & 0.240 & 0.210 \\
\hline CG48 & 38.020 & 23.132 & -34.885 & -14.303 & 0.320 & 0.280 \\
\hline CG49 & 38.097 & 23.213 & -38.434 & -10.379 & 0.470 & 0.450 \\
\hline CG50 & 38.209 & 23.355 & -32.243 & -13.348 & 0.460 & 0.440 \\
\hline CG52 & 38.388 & 23.963 & -30.574 & -15.069 & 0.870 & 0.650 \\
\hline CG53 & 38.230 & 23.854 & -21.086 & -16.692 & 0.350 & 0.340 \\
\hline CG54 & 38.079 & 23.932 & -25.152 & -15.588 & 0.100 & 0.100 \\
\hline CG55 & 38.345 & 24.188 & -30.651 & -14.906 & 0.740 & 0.660 \\
\hline
\end{tabular}




\begin{tabular}{|c|c|c|c|c|c|c|}
\hline SITE & Latitude & Longitude & $\begin{array}{r}\text { Residuals } \\
\text { North } \\
\mathrm{mm} / \mathrm{yr} \\
\end{array}$ & $\begin{array}{r}\text { Residuals } \\
\text { East } \\
m m / y r \\
\end{array}$ & $\begin{array}{c}\sigma-n o r t h \\
m m / y r\end{array}$ & $\begin{array}{c}\sigma \text {-east } \\
\mathrm{mm} / \mathrm{yr}\end{array}$ \\
\hline CG56 & 38.086 & 24.392 & -32.612 & -19.332 & 0.330 & 0.280 \\
\hline CG58 & 38.018 & 23.615 & -24.079 & -22.879 & 0.410 & 0.400 \\
\hline CG61 & 38.014 & 21.580 & -28.914 & -21.542 & 0.450 & 0.520 \\
\hline CG63 & 37.723 & 22.875 & -28.101 & -17.422 & 0.440 & 0.490 \\
\hline CG64 & 37.795 & 22.940 & -33.097 & -17.204 & 0.810 & 0.890 \\
\hline CG65 & 37.804 & 23.093 & -31.207 & -17.095 & 0.300 & 0.270 \\
\hline CG66 & 37.822 & 23.944 & -41.672 & -20.370 & 0.610 & 0.570 \\
\hline CJ00 & 37.861 & 22.737 & -24.054 & -16.810 & 0.340 & 0.290 \\
\hline CK00 & 37.887 & 22.858 & -18.418 & -21.010 & 0.290 & 0.260 \\
\hline СТ00 & 38.376 & 22.392 & -15.842 & -9.211 & 1.090 & 1.090 \\
\hline DE30 & 39.839 & 21.878 & -7.709 & -4.753 & 0.120 & 0.120 \\
\hline DION & 38.079 & 23.933 & -24.780 & -17.665 & 0.100 & 0.100 \\
\hline DIRO & 36.637 & 22.369 & -37.654 & -23.137 & 0.750 & 0.690 \\
\hline DMNA & 37.709 & 23.068 & -31.221 & -15.663 & 0.260 & 0.230 \\
\hline DREP & 38.339 & 21.849 & -13.401 & -0.910 & 0.170 & 0.190 \\
\hline $\mathrm{E} 000$ & 38.191 & 22.102 & -20.994 & -11.634 & 0.270 & 0.290 \\
\hline EG56 & 38.111 & 22.166 & -25.915 & -23.509 & 1.780 & 1.990 \\
\hline EG62 & 38.127 & 22.061 & -20.415 & -28.997 & 1.470 & 1.700 \\
\hline EG75 & 38.152 & 22.135 & -27.873 & -23.109 & 1.650 & 1.680 \\
\hline G000 & 38.066 & 21.950 & -15.614 & -19.492 & 0.250 & 0.230 \\
\hline G104 & 40.159 & 21.359 & -3.569 & -2.843 & 0.110 & 0.110 \\
\hline GOPE & 49.914 & 14.786 & -0.031 & 0.079 & 0.090 & 0.090 \\
\hline GR49 & 40.132 & 21.290 & -3.549 & -2.339 & 0.110 & 0.110 \\
\hline GR69 & 40.184 & 21.498 & -3.400 & -5.338 & 0.120 & 0.110 \\
\hline GRAZ & 47.067 & 15.493 & 0.940 & -0.003 & 0.090 & 0.090 \\
\hline $\mathrm{H} 000$ & 38.529 & 21.980 & -8.971 & -16.182 & 0.360 & 0.330 \\
\hline HERS & 50.867 & 0.336 & 2.554 & 9.548 & 0.090 & 0.100 \\
\hline HFLK & 47.313 & 11.386 & 0.896 & 0.199 & 0.090 & 0.090 \\
\hline HIOS & 38.443 & 26.085 & -18.437 & -17.515 & 0.570 & 0.460 \\
\hline $\mathrm{I} 000$ & 38.445 & 21.903 & -10.160 & -11.466 & 0.450 & 0.340 \\
\hline IERI & 40.436 & 23.848 & -3.169 & -17.053 & 0.930 & 0.720 \\
\hline ILOK & 37.442 & 23.299 & -29.485 & -18.847 & 0.220 & 0.210 \\
\hline IT01 & 38.342 & 22.287 & -14.843 & -15.264 & 1.320 & 1.330 \\
\hline IT23 & 38.407 & 22.303 & -17.568 & -21.590 & 1.420 & 1.470 \\
\hline IT61 & 38.492 & 22.387 & -13.000 & -28.882 & 1.350 & 1.270 \\
\hline JOZE & 52.097 & 21.032 & 0.207 & 0.129 & 0.100 & 0.090 \\
\hline K000 & 38.257 & 21.889 & -21.656 & -11.187 & 0.400 & 0.340 \\
\hline KALI & 40.845 & 22.171 & 9.265 & -0.767 & 0.650 & 0.600 \\
\hline KATV & 35.952 & 27.781 & -28.183 & -12.979 & 0.370 & 0.380 \\
\hline KIT3 & 39.135 & 66.885 & 4.418 & -7.563 & 0.140 & 0.150 \\
\hline KIVE & 37.525 & 22.687 & -37.267 & -13.647 & 0.720 & 0.680 \\
\hline KLKI & 41.009 & 22.833 & 2.845 & -3.887 & 0.840 & 0.660 \\
\hline KLTH & 36.890 & 21.803 & -42.996 & -24.824 & 0.440 & 0.390 \\
\hline
\end{tabular}




\begin{tabular}{|c|c|c|c|c|c|c|}
\hline SITE & Latitude & Longitude & $\begin{array}{r}\text { Residuals } \\
\text { North } \\
\mathrm{mm} / \mathrm{yr}\end{array}$ & $\begin{array}{r}\text { Residuals } \\
\text { East } \\
\mathrm{mm} / \mathrm{yr}\end{array}$ & $\begin{array}{r}\sigma-n o r t h \\
m m / y r\end{array}$ & $\begin{array}{c}\sigma-e a s t \\
m m / y r\end{array}$ \\
\hline KN03 & 40.009 & 21.626 & -11.694 & -4.391 & 0.110 & 0.110 \\
\hline KN29 & 40.072 & 21.721 & -6.322 & -5.159 & 0.110 & 0.110 \\
\hline KN43 & 40.113 & 21.616 & -7.031 & -2.725 & 0.120 & 0.120 \\
\hline KN49 & 40.136 & 21.655 & -5.622 & -4.683 & 0.110 & 0.110 \\
\hline KN88 & 40.207 & 21.587 & -2.355 & -4.156 & 0.120 & 0.120 \\
\hline KOIL & 37.414 & 23.114 & -27.298 & -17.019 & 0.300 & 0.260 \\
\hline KOSG & 52.178 & 5.810 & 1.690 & -0.523 & 0.090 & 0.090 \\
\hline KOSM & 37.087 & 22.737 & -25.950 & -15.671 & 0.320 & 0.270 \\
\hline KRKR & 38.145 & 23.701 & -36.788 & -18.951 & 0.620 & 0.940 \\
\hline KRNA & 39.937 & 22.544 & -12.221 & 0.591 & 0.190 & 0.170 \\
\hline KYLN & 37.941 & 21.136 & -8.808 & 10.075 & 0.760 & 0.650 \\
\hline KYRA & 36.307 & 22.984 & -19.364 & -14.141 & 0.330 & 0.270 \\
\hline L000 & 38.105 & 21.808 & -34.012 & -5.656 & 0.380 & 0.480 \\
\hline LAGA & 40.854 & 23.540 & -3.435 & -9.677 & 0.970 & 0.730 \\
\hline LAMA & 53.892 & 20.670 & 0.334 & 0.472 & 0.100 & 0.080 \\
\hline LEON & 37.184 & 22.823 & -29.538 & -21.048 & 0.250 & 0.250 \\
\hline LEPE & 38.697 & 21.291 & -6.291 & 3.414 & 0.470 & 0.410 \\
\hline LESV & 39.234 & 26.451 & -22.443 & -18.486 & 0.270 & 0.220 \\
\hline LEVK & 38.607 & 22.197 & -14.244 & -9.895 & 0.370 & 0.340 \\
\hline LGRN & 37.677 & 24.000 & -28.621 & -13.394 & 0.570 & 0.500 \\
\hline LI29 & 40.063 & 21.939 & -8.787 & -4.596 & 0.120 & 0.120 \\
\hline LI74 & 40.210 & 21.899 & -7.709 & -3.079 & 0.120 & 0.110 \\
\hline LIMN & 39.851 & 25.126 & -15.451 & -17.129 & 0.360 & 0.280 \\
\hline LIPO & 39.676 & 21.846 & -9.491 & -5.959 & 0.140 & 0.130 \\
\hline LO79 & 40.188 & 22.043 & -7.755 & -4.250 & 0.130 & 0.150 \\
\hline LTSA & 37.955 & 23.996 & -27.806 & -18.708 & 0.810 & 0.680 \\
\hline MADR & 40.429 & 355.750 & 0.832 & -0.729 & 0.090 & 0.120 \\
\hline MAOR & 38.186 & 21.390 & -19.260 & 1.380 & 0.440 & 0.360 \\
\hline MAS1 & 27.764 & 344.367 & 2.900 & -1.705 & 0.090 & 0.150 \\
\hline MATE & 40.649 & 16.704 & 4.725 & -0.318 & 0.090 & 0.100 \\
\hline MDVO & 56.027 & 37.224 & 4.866 & 2.675 & 0.110 & 0.080 \\
\hline MEDI & 44.520 & 11.647 & 2.394 & 2.183 & 0.090 & 0.100 \\
\hline MESS & 38.370 & 21.122 & -11.678 & 0.873 & 0.510 & 0.440 \\
\hline METS & 60.217 & 24.395 & -0.877 & 3.522 & 0.100 & 0.080 \\
\hline MLDR & 37.750 & 22.644 & -17.967 & -8.902 & 0.740 & 0.600 \\
\hline MLSS & 37.946 & 21.352 & -19.705 & -3.540 & 0.510 & 0.470 \\
\hline MLVN & 37.171 & 22.252 & -32.641 & -14.300 & 0.610 & 0.680 \\
\hline MNVS & 36.743 & 23.078 & -35.182 & -18.712 & 0.420 & 0.360 \\
\hline MYRT & 38.075 & 21.504 & -23.379 & -13.383 & 0.970 & 0.920 \\
\hline NEVR & 41.355 & 23.846 & -4.765 & -24.593 & 0.360 & 0.280 \\
\hline $\mathrm{NICO}$ & 35.141 & 33.396 & 4.094 & -10.654 & 0.110 & 0.110 \\
\hline NOTO & 36.876 & 14.990 & 4.108 & -5.258 & 0.090 & 0.110 \\
\hline ONSA & 57.395 & 11.926 & -0.071 & 0.319 & 0.090 & 0.090 \\
\hline
\end{tabular}




\begin{tabular}{|c|c|c|c|c|c|c|}
\hline SITE & Latitude & Longitude & $\begin{array}{r}\text { Residuals } \\
\text { North } \\
\text { mm/yr }\end{array}$ & $\begin{array}{r}\text { Residuals } \\
\text { East } \\
\text { mm/yr }\end{array}$ & $\begin{array}{c}\sigma-n o r t h \\
m m / y r\end{array}$ & $\begin{array}{c}\sigma \text {-east } \\
m m / y r\end{array}$ \\
\hline OREI & 37.347 & 22.656 & -24.290 & -13.432 & 0.540 & 0.530 \\
\hline OXIA & 39.764 & 21.936 & -6.093 & -14.067 & 0.380 & 0.330 \\
\hline P842 & 40.601 & 21.677 & -8.492 & -4.637 & 0.160 & 0.140 \\
\hline PENC & 47.790 & 19.282 & 0.580 & 0.395 & 0.100 & 0.090 \\
\hline PHEL & 37.296 & 23.172 & -32.107 & -14.404 & 0.590 & 0.540 \\
\hline PIGA & 37.065 & 22.911 & -35.862 & -13.655 & 0.530 & 0.490 \\
\hline PLAT & 40.642 & 23.362 & -7.826 & -11.609 & 0.830 & 0.660 \\
\hline PLNI & 37.176 & 22.129 & -21.087 & -20.697 & 0.460 & 0.430 \\
\hline POTS & 52.379 & 13.066 & -0.063 & -0.722 & 0.090 & 0.090 \\
\hline PRST & 36.876 & 22.243 & -35.360 & -19.702 & 0.480 & 0.460 \\
\hline PSMS & 40.373 & 23.444 & -10.691 & -28.530 & 0.160 & 0.140 \\
\hline PYLO & 36.887 & 21.695 & -43.936 & -18.827 & 0.400 & 0.340 \\
\hline RASK & 37.978 & 23.309 & -19.325 & -10.248 & 0.340 & 0.310 \\
\hline RIOE & 38.311 & 21.780 & -9.319 & -15.636 & 0.950 & 2.190 \\
\hline RION & 38.311 & 21.783 & -12.886 & -15.373 & 0.680 & 0.600 \\
\hline ROML & 35.404 & 24.694 & -31.359 & -13.604 & 0.240 & 0.220 \\
\hline SFER & 36.464 & 353.794 & 2.120 & -1.419 & 0.090 & 0.120 \\
\hline SI19 & 40.305 & 21.702 & -5.743 & -4.279 & 0.120 & 0.120 \\
\hline SI34 & 40.343 & 21.562 & -4.349 & -1.461 & 0.120 & 0.110 \\
\hline SI80 & 40.473 & 21.510 & -4.504 & -2.874 & 0.110 & 0.110 \\
\hline SIFB & 40.238 & 21.569 & 3.712 & -5.944 & 0.180 & 0.170 \\
\hline SOFI & 42.556 & 23.395 & -1.902 & -0.787 & 0.100 & 0.100 \\
\hline STHN & 39.994 & 23.919 & -11.378 & -10.284 & 0.120 & 0.110 \\
\hline STRF & 37.069 & 21.866 & -38.403 & -31.661 & 0.880 & 1.100 \\
\hline STRV & 37.098 & 22.409 & -47.130 & -24.686 & 1.230 & 0.970 \\
\hline THEO & 40.964 & 23.120 & 5.852 & -12.235 & 0.880 & 0.850 \\
\hline THIR & 36.346 & 25.439 & -22.615 & -9.052 & 0.390 & 0.510 \\
\hline THOM & 38.392 & 21.488 & -25.462 & -18.061 & 0.720 & 0.680 \\
\hline TOLO & 38.324 & 22.184 & -12.840 & -8.414 & 0.470 & 0.430 \\
\hline TSUK & 40.858 & 21.881 & -4.418 & -13.363 & 0.190 & 0.170 \\
\hline TZIA & 37.623 & 24.368 & -36.845 & -19.679 & 0.590 & 0.610 \\
\hline UPAD & 45.407 & 11.878 & 2.841 & 0.317 & 0.090 & 0.100 \\
\hline VELI & 36.718 & 22.948 & -39.910 & -16.549 & 0.220 & 0.200 \\
\hline VILL & 40.444 & 356.048 & 0.100 & 2.957 & 0.090 & 0.120 \\
\hline VRES & 40.798 & 23.136 & 0.759 & -6.613 & 0.540 & 0.410 \\
\hline VRMS & 37.464 & 23.469 & -31.022 & -22.437 & 0.600 & 0.530 \\
\hline WARE & 50.690 & 5.245 & 1.204 & -1.505 & 0.090 & 0.100 \\
\hline WTZR & 49.144 & 12.879 & 0.173 & -0.260 & 0.090 & 0.090 \\
\hline XRIS & 36.791 & 21.878 & -30.109 & -18.627 & 0.120 & 0.130 \\
\hline YITH & 36.776 & 22.560 & -44.271 & -9.721 & 0.590 & 0.560 \\
\hline ZIMM & 46.877 & 7.465 & 0.859 & -0.414 & 0.090 & 0.100 \\
\hline ZWEN & 55.699 & 36.759 & -0.086 & 4.431 & 0.110 & 0.080 \\
\hline
\end{tabular}


The sites around the Chalkidiki and Grevena area show, in their majority, a very small residual motion of around $10 \mathrm{~mm} / \mathrm{yr}$. Further south there is an obvious SW motion of the sites around Attica, Evvia, east and south Peloponnisos as well as the Aegean Islands. This motion increases from around $\sim 25 \mathrm{~mm} / \mathrm{yr}$ for the Aegean islands to around $30 \mathrm{~mm} / \mathrm{yr}$ near Attica, Evvia and the eastern Peloponnisos coast, and reaches $\sim 40 \mathrm{~mm} / \mathrm{yr}$ for the sites in southern Peloponnisos. This increase has also been suggested previously e.g. Cocard et al. (1999) and Peter et al. (1998). The rapid displacement that is concentrated in the small area of the Corinth and Patras Gulfs is very prominent. The Patras area shows south-westerly motion of $\sim 30-35 \mathrm{~mm} / \mathrm{yr}$.

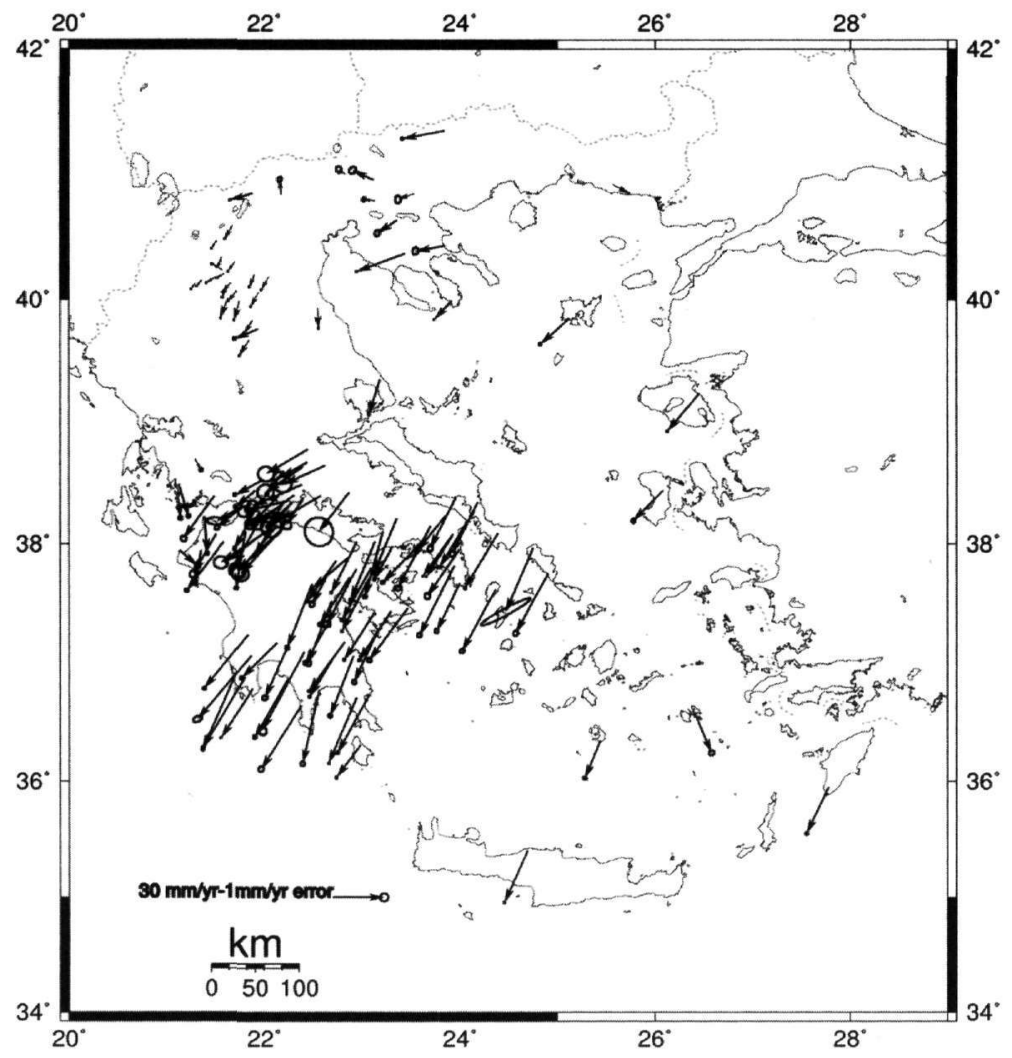

Figure 3 - Velocities for the Greek sites in a Eurasian Fixed reference frame from the period 1994-2000 as formed in this study. $95 \%$ confidence error ellipses are shown

\section{Strain analysis}

The next step of data analysis is to compute geodetic strain-rate tensors and rotation rates by using two different methods. The first is the polygon method which allows us to define the region where the strain is to be computed (e.g. based on geological criteria). The second method of deriving strain rates calculates the stain by applying a continuous curvature gridding to the horizontal velocity field data, and is independent of geological preferences and allows interpolation between points.

For the first method the area is divided up into polygonal regions bounded by geodetic monuments. The strain rate is assumed to be uniform within each polygonal region and constant with respect with time. No assumptions are made regarding the continuity of velocity or strain rate between regions (Frank 1966, Welsch 1983). The choice of the polygons is very important but somewhat arbitrary although reasonable criteria can be adopted. The Greek area is quite compli- 
cated because it contains many active faults, most of which appear to have segments of around 15$20 \mathrm{~km}$ in length. Primarily we created the smallest possible polygons according to major tectonic zones as defined by previous researchers (Kahle et al. 1998, Le Pichon and Angelier 1979, Le Pichon et al. 1995, Papazachos et al. 1999). Following this step, neighbouring polygons with similar strain pattern are merged. We tried to ensure that the final polygons bound the whole of either main fault zones or trough zones. That was not always possible because the faults can be quite small or concentrated in a narrow area (e.g. Corinth Gulf, Evvia Gulf). In the final polygons the uniform strain is in a good fit (root mean square weighted residual applied) to all the stations that pertain to it. In total 22 polygons were formed. The principal horizontal strain rates, for each of the chosen 22 polygons, are computed using the program polystr 2 (Clarke 1996). From the velocity gradient tensor the strain parameters and their formal errors are derived. Table 4 and Figure 4 summarise the results for each polygon. There is generally a large uncertainty in the azimuth of the principal strain rates, although their magnitudes are relatively certain. Figure 5 presents the geodetic rigid body rotation for each of the 22 polygons. Strong clockwise rotation is observed around the North Eastern Aegean, Gulf of Corinth, and Gulf of Evvia, with smaller rates around the Grevena and Chalkidiki areas. The sense of rotation changes in the South Aegean and Cyclades area where anti-clockwise rotation is present.

Table 4 - Principal horizontal strain rates (ppm/year), azimuth of most compressive principal strain (degrees), and rates of rotation and dilatation (ppm/year)

\begin{tabular}{|c|c|c|c|c|c|c|c|c|c|c|}
\hline Polygon & $\begin{array}{c}\text { Eps1 } \\
(\mathrm{ppm} / \mathrm{yr})\end{array}$ & 16 & $\begin{array}{c}\text { Eps2 } \\
(\mathrm{ppm} / \mathrm{yr})\end{array}$ & $1 \sigma$ & $\begin{array}{l}\text { Azim } \\
\text { (deg) }\end{array}$ & $1 \sigma$ & $\begin{array}{c}\text { Rotation } \\
\text { (deg/Myr) }\end{array}$ & $1 \sigma$ & $\begin{array}{c}\text { Dilatation } \\
\text { (ppm/yr) }\end{array}$ & $1 \sigma$ \\
\hline 1 & 0.174 & 0.006 & -0.037 & 0.005 & -86.9 & 1.50 & 6.6 & 0.22 & 0.138 & 0.008 \\
\hline 2 & 0.108 & 0.004 & 0.019 & 0.005 & -75.5 & 3.61 & -0.3 & 0.17 & 0.127 & 0.006 \\
\hline 3 & 0.072 & 0.008 & -0.019 & 0.006 & -68.5 & 8.40 & 10.5 & 0.29 & 0.054 & 0.010 \\
\hline 4 & 0.281 & 0.010 & 0.057 & 0.009 & -79.1 & 2.81 & -6.7 & 0.39 & 0.338 & 0.013 \\
\hline 5 & 0.092 & 0.007 & -0.179 & 0.019 & 86.7 & 3.44 & 2.1 & 0.65 & -0.087 & 0.020 \\
\hline 6 & 0.072 & 0.002 & -0.125 & 0.004 & 75.9 & 1.11 & 0.6 & 0.12 & -0.053 & 0.119 \\
\hline 7 & 0.197 & 0.005 & -0.214 & 0.005 & 85.7 & 0.66 & 2.2 & 0.19 & -0.017 & 0.007 \\
\hline 8 & 0.261 & 0.008 & -0.013 & 0.024 & 78.2 & 4.53 & 2.5 & 0.73 & 0.248 & 0.025 \\
\hline 9 & 0.021 & 0.026 & -0.129 & 0.071 & -36.1 & $\begin{array}{r}50.2 \\
1\end{array}$ & 11.1 & 2.16 & -0.108 & 0.076 \\
\hline 10 & 0.113 & 0.014 & 0.004 & 0.009 & -4.5 & 6.20 & 8.0 & 0.47 & 0.117 & 0.017 \\
\hline 11 & 0.039 & 0.004 & -0.018 & 0.004 & 1.5 & 4.56 & 1.9 & 0.18 & 0.020 & 0.006 \\
\hline 12 & 0.143 & 0.007 & -0.074 & 0.005 & 75.5 & 2.02 & 3.2 & 0.23 & 0.069 & 0.008 \\
\hline 13 & 0.023 & 0.003 & -0.045 & 0.003 & 50.1 & $\begin{array}{r}43.9 \\
9 \\
\end{array}$ & 3.6 & 0.11 & -0.022 & 0.003 \\
\hline 14 & 0.080 & 0.002 & -0.043 & 0.001 & -76.2 & 1.04 & 1.2 & 0.07 & 0.037 & 0.003 \\
\hline 15 & 0.095 & 0.004 & 0.001 & 0.002 & -64.3 & 4.87 & -2.5 & 0.13 & 0.096 & 0.005 \\
\hline 16 & 0.003 & 0.002 & -0.164 & 0.008 & 27.3 & 5.83 & -1.5 & 0.23 & -0.161 & 0.008 \\
\hline 17 & 0.091 & 0.004 & -0.011 & 0.001 & -45.2 & $\begin{array}{r}50.7 \\
8\end{array}$ & -1.9 & 0.11 & 0.079 & 0.004 \\
\hline 18 & 0.093 & 0.005 & -0.013 & 0.003 & -78.6 & 2.72 & 1.6 & 0.18 & 0.080 & 0.005 \\
\hline 19 & 0.135 & 0.014 & -0.449 & 0.009 & 0.5 & 1.15 & -2.2 & 0.47 & -0.314 & 0.016 \\
\hline 20 & 0.069 & 0.001 & -0.052 & 0.001 & 62.0 & 1.84 & 1.8 & 0.05 & 0.017 & 0.002 \\
\hline 21 & 0.113 & 0.003 & -0.038 & 0.001 & 82.6 & 0.80 & -1.4 & 0.07 & 0.075 & 0.003 \\
\hline 22 & 0.166 & 0.002 & -0.008 & 0.001 & -42.9 & $\begin{array}{r}48.7 \\
0\end{array}$ & 6.3 & 0.07 & 0.174 & 0.003 \\
\hline
\end{tabular}




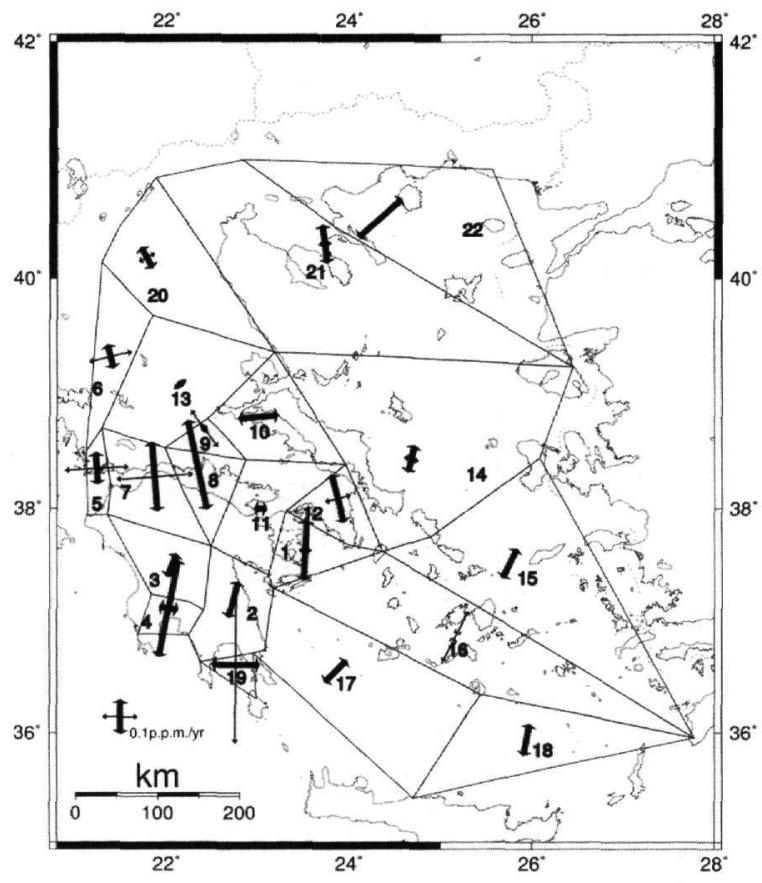

Figure 4 - Principal strain rates computed for the 22 polygonal regions. Extensional axes are shown as thick lines and compressional axes as shown as thin lines. Results are given in $\mathbf{p p m} / \mathbf{y r}$

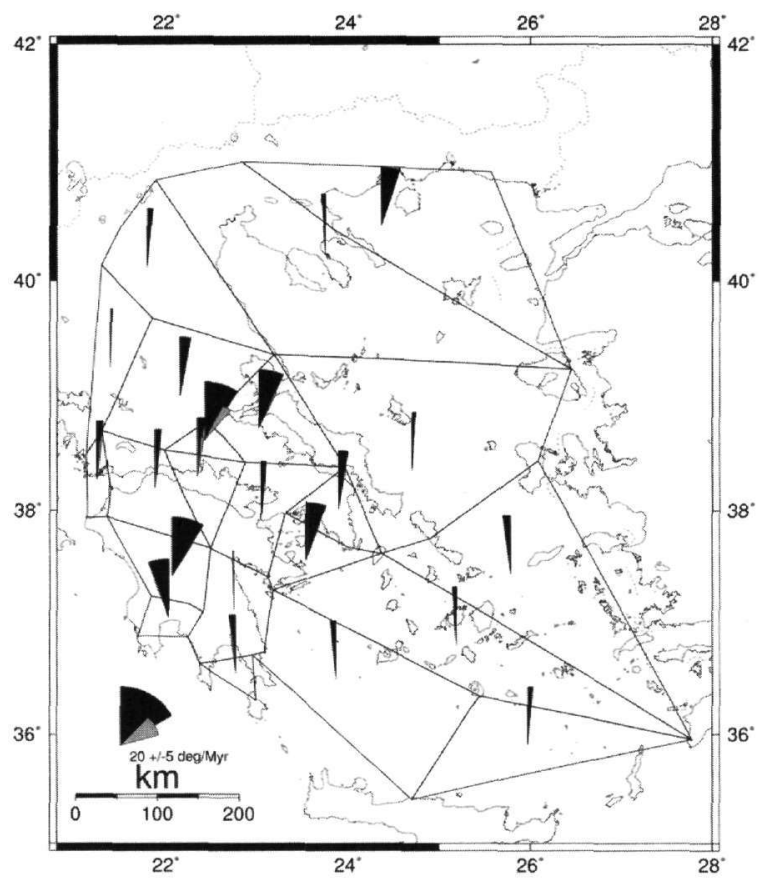

Figure 5 - Geodetic rigid-body rotation rates computed from the polygonal regions (deg/Myr). Uncertainties (95\% confidence) are shown in grey 
The second method of deriving horizontal geodetic strain rates is independent of geological preferences, depending only on the velocity field data. We applied a continuous curvature gridding to the horizontal velocity field data allowing interpolation between points. Three different grid spacing values were tested $(10,30,40$ minutes) with the 30 minute grid spacing chosen. The 10 minute grid did not show any regions of different strain patterns when compared with the 30 minute grid, whereas the 40 minute grid masked detail that was observed with the 30 minute grid. The principal strain rates from the above are shown in Figure 6.

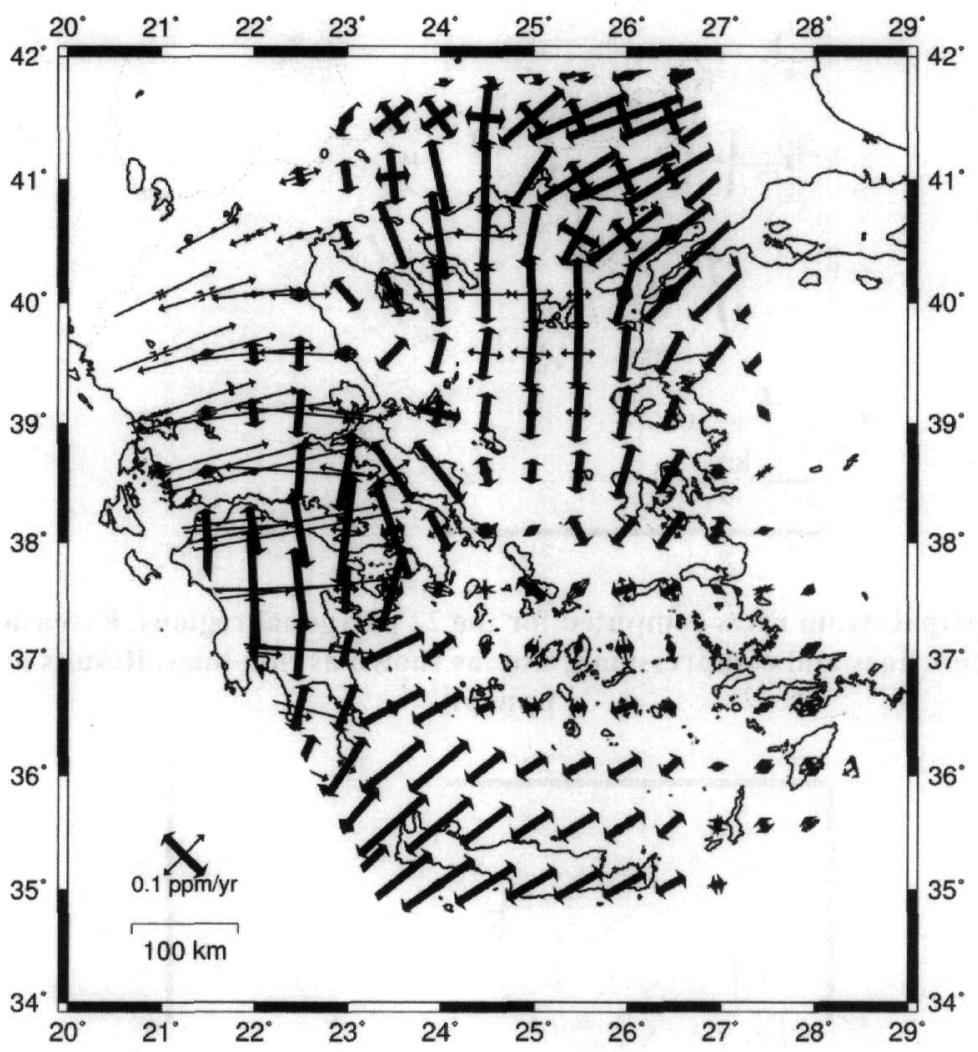

Figure 6 - Principal strain rates computed from the minimum curvature grid method

There is a very good agreement when comparing strain rates between the grid and polygon methods. In general the Hellenic area experiences a rapid extensional deformation. This model shows areas bounded by fault systems e.g. Gulf of Evvia, Gulf of Corinth, and Gulf of Messinia undergoing rapid extension. The central and southern Aegean are extending. The region around the Cyclades is almost strain free. In western Greece where continental collision takes places, rapid compression is present increasing in the Gulf of Patras. Differences are observed near to the edges of the grid (e.g. Laconia Gulf and western Greece) where there are too few data points for the interpolation method to be reliable and spurious effects occur. Agreement is observed in north Greece (Grevena area), north Aegean, Corinth Gulf area, Patras Gulf area, western Peloponnisos, south Aegean (Sea of Crete). An obvious difference is observed around the northern Gulf of Evvia. In the polygon method (polygon 10, Fig. 4) the direction of deformation is E-W, whereas in the gridding methods the direction of deformation is NNW-SSE. However, in the polygon method the azimuth has a large uncertainty because only five relatively closely-spaced data points are used, whereas in the gridding methods data from the wider region will have an influence. In both methods the deformation is extension at $\sim 0.11 \mathrm{ppm} / \mathrm{yr}$. The geodetic-rigid body rotation rates show a 
similar pattern for all the methods applied. Strong clockwise rotation is observed in the Peloponnisos area, Central Greece and north Aegean Sea, while rapid counter-clockwise rotation is observed in the central Aegean, Cyclades and Sea of Crete.

\section{Conclusion}

GPS measurements from $1994-2000$ were reprocessed in order to determine the magnitude and style of deformation throughout the Hellenic region, in a Europe-fixed reference frame. Results from previous studies cannot be rigorously combined because the reference frame constraints and the conventions of the processing software used cannot be removed using the available information. 18 GPS campaigns from $1994-2001$ covering 280 local sites have been included, with a reference frame navigated by 43 global, long-running IGS sites. Using the Altamimi et al. (2002) European absolute Euler pole, a solution is derived in a Europe-fixed reference frame. In the Hellenic region, sites around the Chalkidiki and Grevena areas show a small residual motion of around $10 \mathrm{~mm} / \mathrm{yr}$ with respect to stable Europe. Sites around Attica, Evvia, and the eastern and southern Peloponnisos, as well as the Aegean Islands, have a SW motion which increases from $\sim 25 \mathrm{~mm} / \mathrm{yr}$ for the Aegean islands to around $30 \mathrm{~mm} / \mathrm{yr}$ around Attica, Evvia and the eastern Peloponnisos coast and $\sim 40 \mathrm{~mm} / \mathrm{yr}$ for sites in the southern Peloponnisos. The Patras area shows a residual motion of around $30-35 \mathrm{~mm} / \mathrm{yr}$.

Geodetic strain-rate tensors and rotation rates are computed firstly within independent contiguous polygons, and secondly by applying continuous curvature gridding to the horizontal velocity field data. The two methods give very similar results. In general terms the Hellenic area is a region undergoing a rapid extensional deformation. Areas bounded by fault systems e.g. Gulf of Evvia, Gulf of Corinth, and Gulf of Messinia undergo strong extension. The central and south Aegean are also extending but the Cyclades area is almost strain free. In western Greece where continental collision takes place, strong compression is present, increasing in Gulf of Patras. Clockwise rotation is observed in Central Greece around the Gulfs of Corinth and Evvia, turning to anticlockwise rotation in the south Aegean and South Peloponnisos.

\section{References}

Altamimi, Z., Sillard, P., and Boucher, C., 2002. ITRF2000: A new release of the International Terrestrial Reference Frame for earth science applications, J.Geophys.Res, 107(B10), 2214. doi:10.1029/2001JB000561

Ambraseys, N., 2001. Reassessment of earthquakes,1900-1999, in the Eastern Mediterranean and the Middle East, Geophys.J.Int, 145, 471-485.

Ambraseys, N., and Jackon, J., 1997. Seismicity and strain in the Gulf of Corinth (Greece) since 1694, J.Earthq. Eng, 1(3), 433-474.

Ambraseys, N., and Jackson, J., 1990. Seismicity and associated strain of central Greece between 1890 and 1988, Geophys.J.Int, 101, 663-708.

Armijo, R., Meyer, B., King, G.C.P., Rigo, A., and Papanastasiou, D., 1996. Quaternary evolution of the Corinth Rift and its implications for the Late Cenozoic evolution of the Aegean, Geophys.J.Int, 126, 11-53.

Beutler, G., et al., 2001. Bernese GPS software Version 4.2, Astronomical Institute, University of Berne 2001.

Briole, P., et al., 2000. Active deformation of the Corinth rift, Greece: Results from repeated Global Positioning System surveys between 1990 and 1995, J.Geophys.Res, 105(B11), 25.605-25.625. 
Clarke, P.J., 1996. Tectonic motion and tectonic deformation in Greece from GPS measurements, University of Oxford, Exeter College, Oxford.

Cocard, M., et al., 1999. New constraints on the rapid crustal motion of the Aegean region: recent results inferred from GPS measurements (1993-1998) across the West Hellenic Arc, Greece, Earth Planet.Sci.Lett, 172, 39-47.

Cruddace, P.R., et al., 1999. An interdisciplinary approach to studying seismic hazard throughout Greece. In Geodesy beyond 2000: the challenges of the first decate, International Association of Geodesy Symposia, 121, 279-284.

Davies, P., 1997. Assembling the IGS polyhedron. a densinfied weekly GPS terrestrial reference frame, University of newcastle Upon Tyne, Newcastle Upon Tyne.

Davies, R.R., and Blewitt, G., 2000. Methodology for global geodetic time series estimation: A new tool for geodynamics, J.Geophys.Res, 105(B5), 11.083-11.100.

Estay, L., and Meertens, C., 1999. TEQC: The Multi-Purpose Toolkit for GPS/GLONASS data, GPS solutions, 3(1): 42-42.

Frank, F.C., 1966. Deduction of earth strains from survey data, Bull.Seismol.Soc.Am, 56(1), 32-42.

Goldsworthy, M., Jackson, J., and Hains, A.J., 2002. The continuity of the active fault systems in Greece, Geophys.J.Int, 148, 596-618.

Gurtner, W., 1994. RINEX: The Receiver Independent Exchange Format, GPS WORLD, 5(7): 4852.

Hatzfeld, D., Martinod, J., Bastet, G., and Gautier, P., 1997. An analogue experiment for the Aegean to describe the contribution of gravitational potential energy, J.Geophys.Res, 102, 649-659.

Hollenstein, C., Geiger, A., Kahle, H.-G., and Veis, G., 2005. CGPS time-series and trajectories of crustal motion along the West Hellenic Arc, Geophys.J.Int, 164, 182-191. doi:10.1111/j.1365-246X.2005.02804.X

Kahle, H.-G., et al., 2000. GPS-derived strain rate field within the boundary zones of the Eurasian, African, and Arabian Plates, J.Geophys.Res, 105(B10), 23.353-23.370.

Kahle, H.-G., et al., 1999. The GPS strain rate field in the Aegean Sea and western Anatolia, Geophys.Res.Lett, 26(16), 2513-2516.

Kahle, H.-G., et al., 1998. The strain rate field in the eastern Mediterranean region estimated by repeated GPS measurements, Tectonophysics, 294, 237-252.

Kreemer, C., Holt, W.E., and Hains, A.J., 2003. An integrated global model of present-day plate motions and plate boundary deformation, Geophys.J.Int, 154, 8-34.

Lavallée, D.A., 2000. Tectonic plate motions from global GPS measurements, University of Newcastle Upon Tyne, Newcastle Upon Tyne.

Le Pichon, X., and Angelier, J., 1979. The Hellenic arc and trench system: a key to the evolution of eastren Medoterranean, Tectonophysics, 60, 1-42.

Le Pichon, X., Chamot-Rooke, N., and Lallemant, S., 1995. Geodetic determination of the kinematics of central Greece with respect to Europe: Implications for eastern Mediterranean tectonics, J.Geophys.Res, 100(B7), 12.675-12.690.

Mantovani, E., et al., 2000. Role of kinematically induced horizontal forces in Mediterranean tectonics: insight from numerical modelling, J.Geodyn, 30, 287-320. 
McClusky, S., et al., 2000. Global Positioning System Constraints on plate kinematics and dynamics in the eastren Mediterranean and Caucasus, J.Geophys.Res, 105(B3), 5695-5719.

McKenzie, D.P., 1970. Plate Tectonics of the Mediterranean Region, Nature, 226, 239-243.

McKenzie, D.P., 1972. Active tectonics of the Mediterranean Region, Geophys.J.R.astr.Soc, 30, 109-185.

McKenzie, D.P., 1978. Active tectonics of the Alpine-Himalayan Belt: the Aegean Sea and surrounding regions, Geophys.J.R.astr.Soc, 55,: 217-254.

McKenzie, D.P., and Jackson, J., 1983. The relationship between strain rates, crustal thickening, paleomagnetism, finite strain and fault movements within a deforming zone, Earth Planet.Sci.Lett, 65, 182-202.

McKenzie, D.P., and Jackson, J., 1986. A block model of distributed deformation by faulting, J.Geol.Soc.London, 143, 349-353.

Meijer , P.T., and Wortel, M.J.R., 1997. Present-day dynamics of the Aegean region: a model analysis of the horizontal pattern of stress and deformation, Tectonics, 16, 879-895.

Niell, A.E., 1996. Global mapping functions or the atmosphere delay at radio wavelengths, J.Geophys.Res, 101(B2), 3227-3246.

Nyst, M.C.J., and Thatcher, W., 2004. New constraints on the active tectonic deformation of the Aegean, J.Geophys.Res, 109, B11406. doi:10.1029/2003JB002830

Papazachos, B.C., 1990. Seismicity of the Aegean and the surrounding area, Tectonophysics, 178, 287-308

Papazachos, B.C., Papaioannou, C., Papazachos, C.B., and A.S, S., 1999. Rupture zones in the Aegean region, Tectonophysics, 308, 205-221.

Papazachos, C.B., and Kiratzi, A., 1996. A detailed study of the active crustal deformation in the Aegean and surrounding area, Tectonophysics, 253, 129-153.

Peter, J.D., et al., 1998. Establishment of continuous GPS network across Kephalonia Fault Zone, Ionian islands, Greece, Tectonophysics, 294, 253-260.

Plag, H.-P., et al., 1998. Scientific objectives of current and future WEGENER activities, Tectonophysics, 294, 177-223.

Reilinger, R., et al., 1997. Global Positioning System measurements of present-day crustal mmovements in the Arabia-Africa-Eurasia plate collision zone, J.Geophys.Res, 102(B5), 9983-9999.

SOPAC, Scripps Orbit and Permanent Array Center

http://sopac.ucsd.edu/dataArchive/: (downloaded, May 2006)

Taymaz, T., Jackson, J., and McKenzie, D.P., 1991. Active tctonics of the north and central Aegean Sea, Geophys.J.Int, 106, 433-490.

Thatcher, W., 2003. GPS Constraints on the Kinematics of Continental Deformation, Internat.Geology Review, 45, 191-212.

UNAVCO, TEQC-tutorial. http://facility.unavco.org/software/teqc/teqc.html: (downloaded, May 2006)

Welsch, W.M., 1983. Finite element analysis of strain patterns from geodetic observations across a plate margin, Tectonophysics, 97, 57-71. 